\title{
The Effective Attempt of the Course for Applications of Resonance
}

\author{
Jianjun Yin \\ School of information science and technology \\ Fudan University \\ Shanghai, China \\ yinjianjun@fudan.edu.cn
}

\begin{abstract}
In this paper, we made a good attempt for the application of resonance of the college circuit theory course, including the review of resonant knowledge, two resonant applications, an experiment of radio antenna tuning loop, the discussion of the impact of the $Q$ value with improvements and the further applications of resonance.
\end{abstract}

Keywords-education; Effective attempt; resonance; series resonance; parallel resonance

\section{REVIEW OF RESONANT KNOWLEDGE}

At the beginning of the course, we give a review of the series and parallel resonance of the last course, including the vector diagram of voltage and current, the resonance angle frequency and the quality factor, etc. [1, 2]. We also summarize the characteristics of series resonance: the resistance reaches the minimum, the current maximum, the capacitor and the inductor can obtain a resonance voltage which is much higher than that of the driving voltage (Q times) [3, 4].

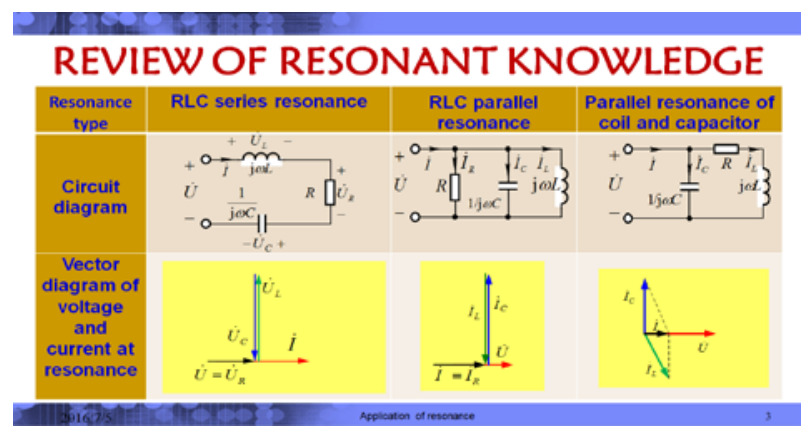

Fig. 1. Course review 1

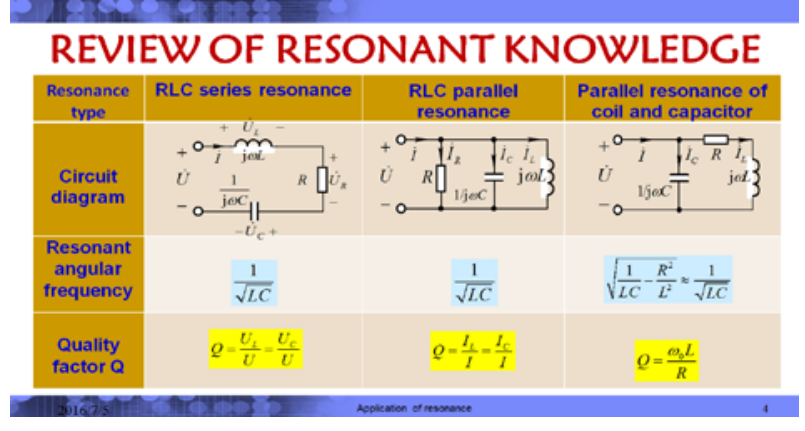

Fig. 2. Course review 2

\section{INTRODUCTION OF RESONANT APPLICATIONS}

We know that in telecommunication engineering, the output of some signal source is very weak! Can we use the resonance to amplify and select the signal we are interested in it? We begin by discussing the example of a radio. When the dial knob is rotated to a certain position, the corresponding frequency of the radio sound come out, we say that the radio is the application of the principle of resonance.

\section{INTRODUCTION OF RESONANT APPLICATIONS}
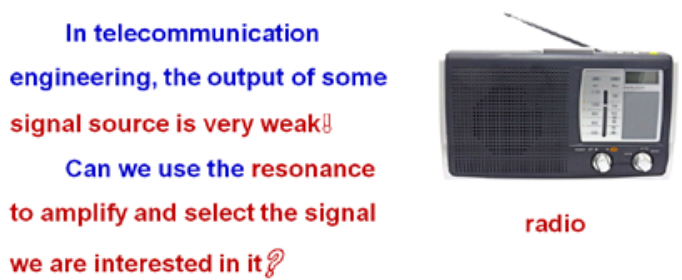

radio

Fig. 3. Introduction of resonant application

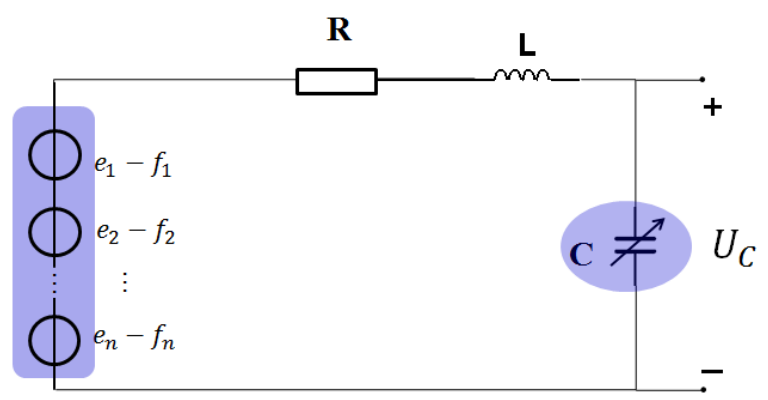

Fig. 4. Equivalent circuit diagram of the radio 1

Fig. 4 is the equivalent circuit diagram of the radio antenna tuning and receiving circuit. This is a RLC series circuit, where $\mathrm{e} 1, \mathrm{e} 2 \ldots$ and en represent the voltage of each frequency induced by the receiving end, representing the different frequencies of the radio signal. All of these signals are very weak. The equivalent circuit of the coil of the receiving end is expressed by $\mathrm{R}$ and $\mathrm{L}$. The output is the voltage of the capacitance $\mathrm{C}$. In fact, the adjustment of the selector knob is to 
adjust the size of the capacitor $\mathrm{C}$, which makes the circuit of the radio resonance. Then according to the relevant knowledge of RLC series resonance, we can obtain a resonance voltage which is much higher than that of the driving voltage ( $Q$ times). The other frequency signals, since they are not at the resonant frequency, the impedance is large, the current is smaller, thus the voltage obtained from the variable capacitance is relatively low, so we can hear only the voice at resonant frequencies.

Suppose that there are three frequencies of radio signals, that is, e1, e2, e3, the corresponding frequencies are $648 \mathrm{kHz}$, $855 \mathrm{kHz}$ and $990 \mathrm{kHz}$, respectively, the effective value of the induction voltage are $15 \mathrm{uV}$, and in the circuit $\mathrm{R}=33 \mathrm{ohm}$, $\mathrm{L}=330 \mathrm{uH}$. In order hear the $990 \mathrm{kHz}$ radio program, according to the resonance formula, we have that $\mathrm{C}$ approximately equals to 78.3pf.

At this point, we can calculate that the effective values of the voltages at both ends of the capacitor $\mathrm{C}$ at $648 \mathrm{kHz}, 855 \mathrm{kHz}$ and $990 \mathrm{kHz}$ are respectively $26.2 \mathrm{uV}, 58.9 \mathrm{uV}$ and $933 \mathrm{uV}$. We can see that at frequency $990 \mathrm{kHz}$, we get $933 \mathrm{uV}$, which amplifies about 62 times the original. Although the other two radio signal also be amplified, their amplitude is far smaller than that of the $990 \mathrm{kHz}$ signal, so we have only heard $990 \mathrm{kHz}$ 's radio program.

Students can also try to calculate the strength of the radio signal when the capacitor is adjusted to make the circuit resonate at $648 \mathrm{kHz}$ or $855 \mathrm{kHz}$. In addition, please think about that whether you can choose the station by adjusting the inductor L? The answer is yes, as shown in Fig. 5; the students can refer to the above examples to do some calculations after class.

The radio antenna tuning and receiving circuit

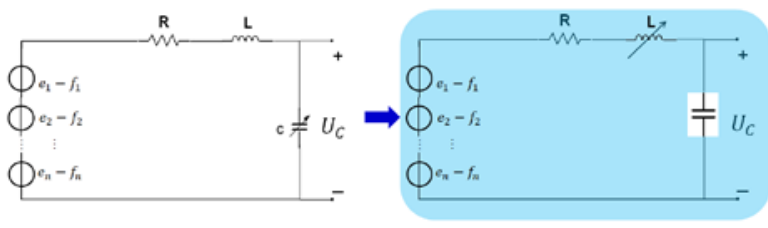

Fig. 5. Equivalent circuit diagram of the radio 2

\section{RADIO ANTENNA TUNING LOOP - EXPERIMENT}

We further deepen our understanding through an experiment. We follow the above circuit diagram; the devices are inserted on the circuit board. Assume that there are two radio signals at $158 \mathrm{kHz}$ and $300 \mathrm{kHz}$. The $158 \mathrm{kHz}$ signal is produced by the experimental circuit board and the $300 \mathrm{kHz}$ signal is generated by the signal generator, the peak values are both $0.1 \mathrm{~V}$.

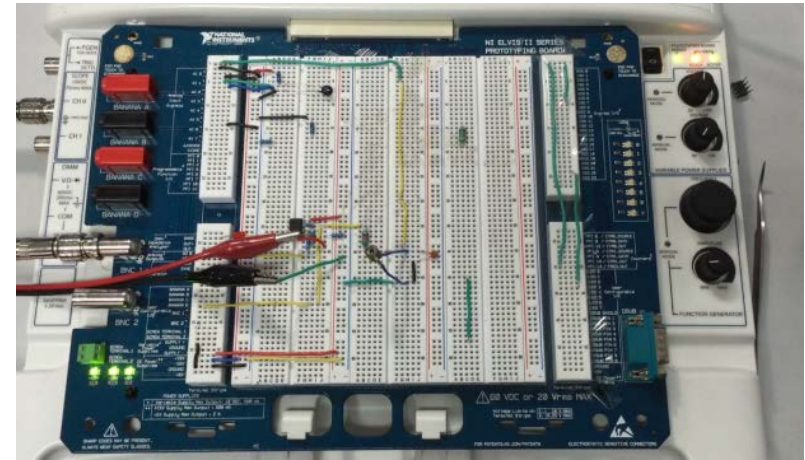

Fig. 6. Experimental circuit board

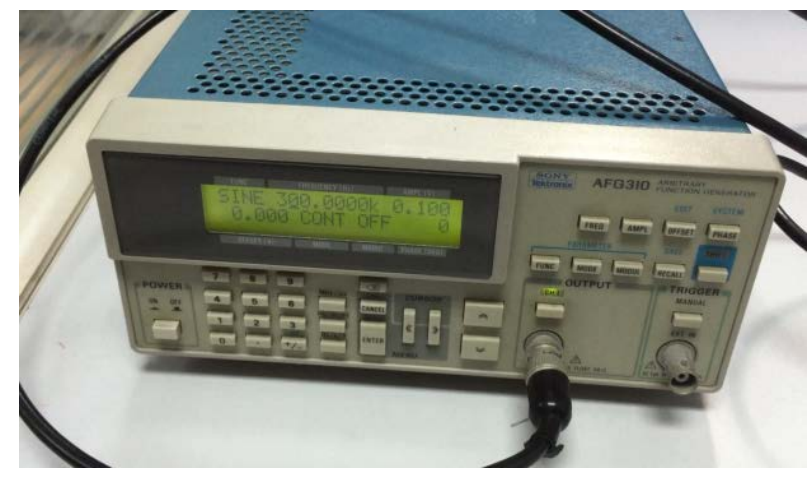

Fig. 7. Signal generator

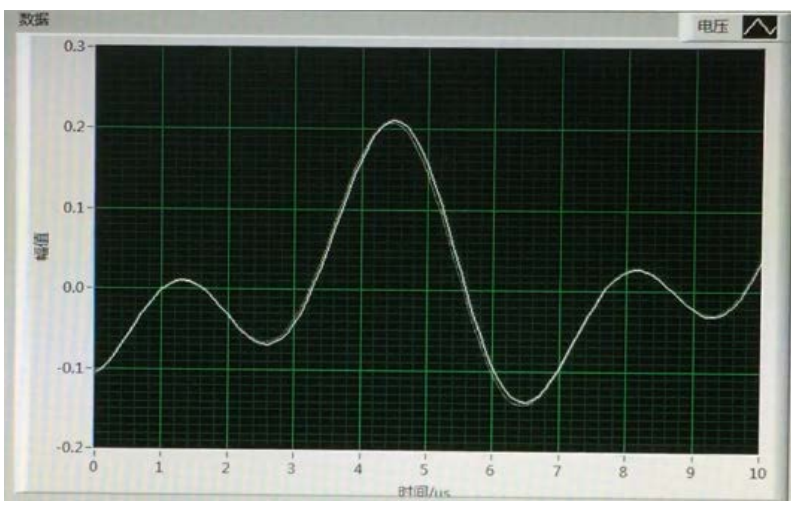

Fig. 8. Mixed waveform of the input signals

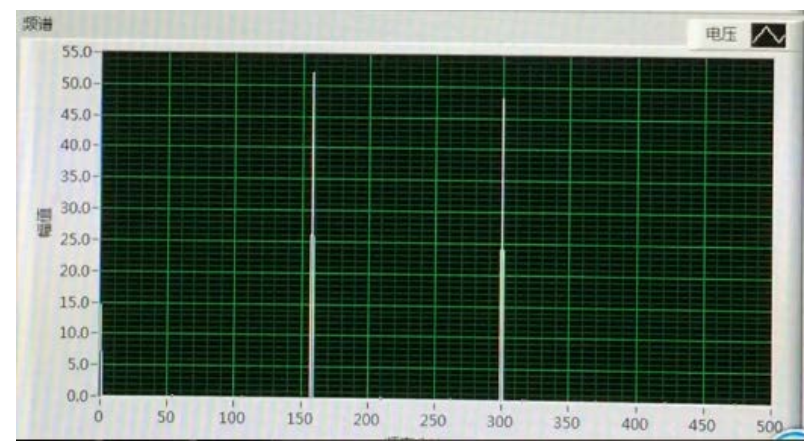

Fig. 9. Spectrum map of the input signals 
As shown in Fig. 8, the mixed waveform of the input $158 \mathrm{kHz}$ and $300 \mathrm{kHz}$ signal is more messy, and we can see from the spectrum map that the magnitudes of the two waveforms are close. Then we adjust of the capacitor $C$ so that the signal of $158 \mathrm{kHz}$ achieves resonance. Let's take a look at the voltage of the capacitor again.

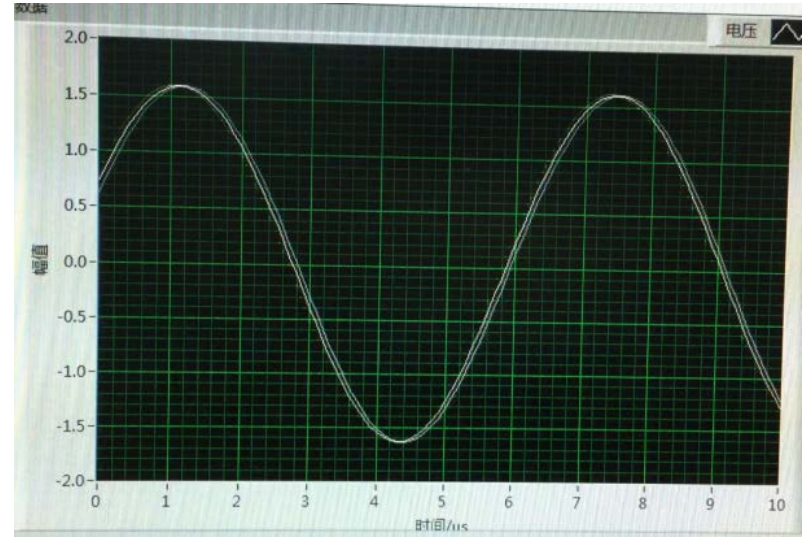

Fig. 10. The output signal

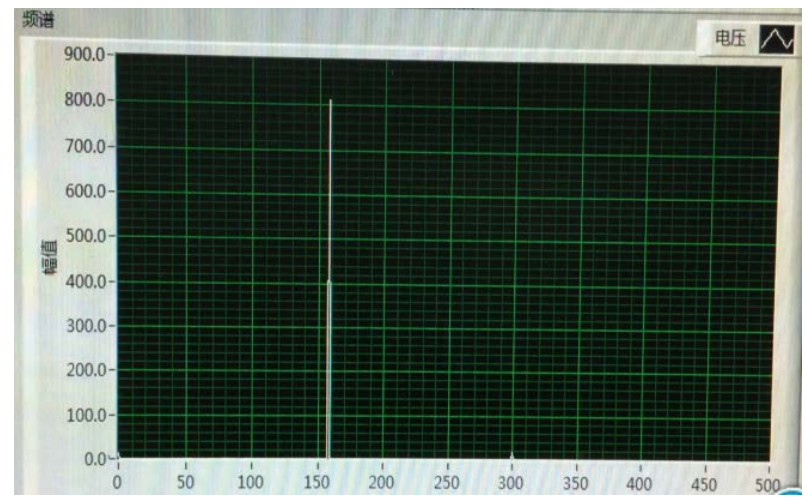

Fig. 11. Spectrum map of the output signal

As shown in Fig. 10, we can see that relative to the input signal, the output is more stable. We can only see the $158 \mathrm{kHz}$ signal and the peak is about $1.6 \mathrm{~V}$, which is much higher than the input peak. Also from the spectrum graph we can see that as compared with the $158 \mathrm{kHz}$ signal, the $300 \mathrm{kHz}$ signal is almost negligible.

By this experiment, we further verify the effect of the resonance; we get the signal at the output of the signal with much higher amplitude than the input signal at the output.

\section{FLUORESCENT LAMP START}

Here we look at the phenomenon when the fluorescent lamp is lit. Generally fluorescent lamp needs thousands of volts or more to be lit, this voltage is far more than the power supply voltage of our family. Then how can we light the fluorescent lamp? Can the principle of resonance be applied? Yes !

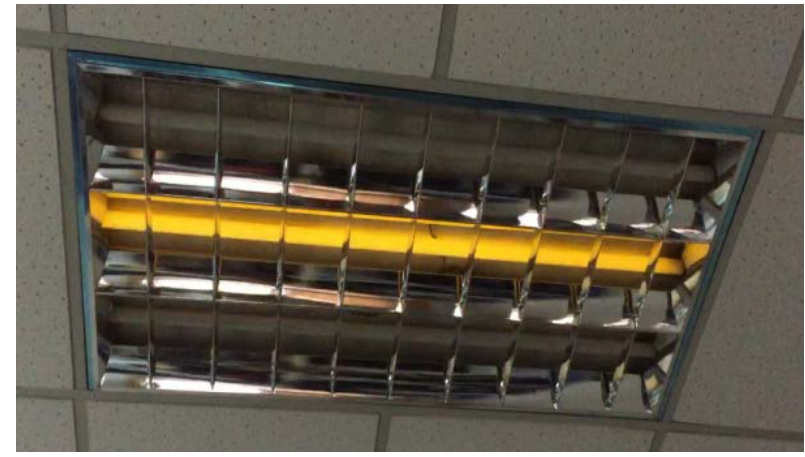

Fig. 12. Light up the fluorescent lamp 1

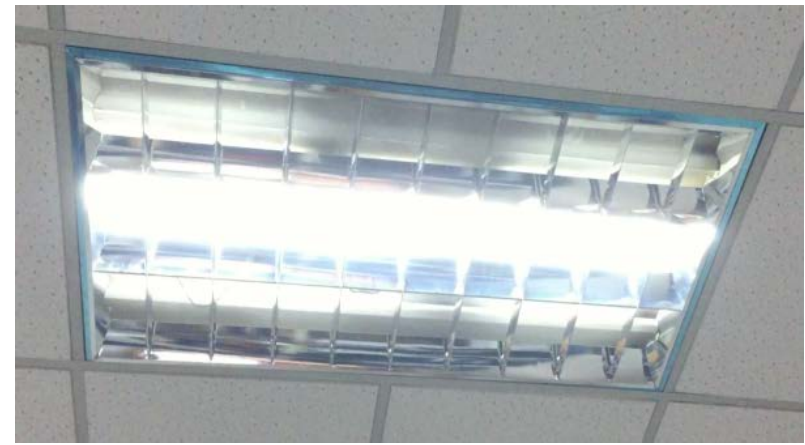

Fig. 13. Light up the fluorescent lamp 2

Fig. 14 shows the equivalent circuits for lighting up the fluorescent lamp, where an electronic ballast integrated circuit chip and the external capacitance $\mathrm{C}$ and inductance $\mathrm{L}$ are used. The fluorescent lamp is connected with the two ends of the capacitor $\mathrm{C}$. The chip adjusts the frequency by using sweep mode. Even if the $\mathrm{L}$ and $\mathrm{C}$ have relatively large errors, it can always find the resonance point, when the voltage. At this time, the voltage of the $\mathrm{C}$ can reach the starting voltage of the fluorescent lamp, so as to light the fluorescent lamp.

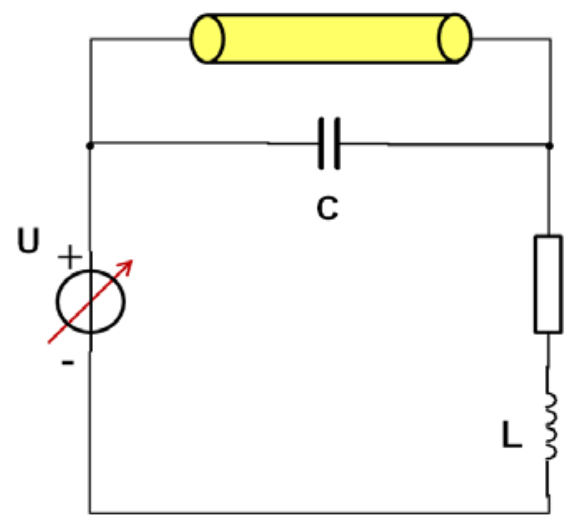

Fig. 14. Equivalent circuit for lighting up the fluorescent lamp 


\section{DISCUSSION- THE MAIN FACTORS AFFECTING THE Q VALUE}

Those who have done the experiment may find a phenomenon that the $\mathrm{Q}$ values obtained in the experiments are often smaller than those calculated theoretically. What is the reason? Fig. 15 shows the brief equivalent diagram of the resonant circuit of the intermediate frequency amplifier for Heterodyne receiver.

\section{DISCUSSION-THE MAIN FACTORS AFFECTING THE Q VALUE}

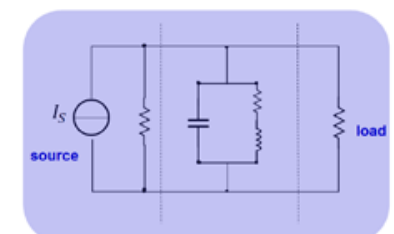

Q - actual value smaller

than the theoretical one ?

- The source is NOT ideal

- The load is not ideal

- Loop resistance etc.

The diagram of the resonant circuit of the intereediat

frequency alaplifier for Heterodyne receiver

Fig. 15. The main factors affecting the $Q$ value

Firstly we often assume that theoretically the circuit of the signal source is ideal, the series resistance of the voltage source is 0 , and the shunt resistance of the current source is infinity. However, in fact, as shown in Fig. 15. , as the driving source of intermediate frequency amplifier, the shunt resistance of the transistor collector is a finite value, which is generally dozens of $\mathrm{K} \Omega$.

Secondly, we often do not consider the impact of the load of the circuit, but this is not correct in practice. As shown in Fig. 10, the input resistance of the load is not infinite; also the amplifier's secondary load is usually only a few $\mathrm{k} \Omega$.

In addition, in theory, we often do not consider the resistance of the LC circuit. In fact, there are indeed, particularly the resistance of the coil can't be avoided.

So how can we reduce the impact of the $\mathrm{Q}$ value? According to the previous factors, considering that the loop resistance is not easy to remove, we can improve it from reducing the influence of the source resistance and load impedance. As shown in Fig. 11, we can introduce the transformer and tap to improve the impact of the circuit. Assuming that the ratio of primary coil and secondary coil are shown in Fig. 11, the original source resistance and load resistance is constant. Then according to the resistance transformation of transformer, the source resistance will increase 4 times as much as that of the original's, and load resistance will increase 400 times as much as that of the original's, which greatly reduces the influence.

\section{DISCUSSION-REDUCING THE IMPACT OF THE Q VALUE?}

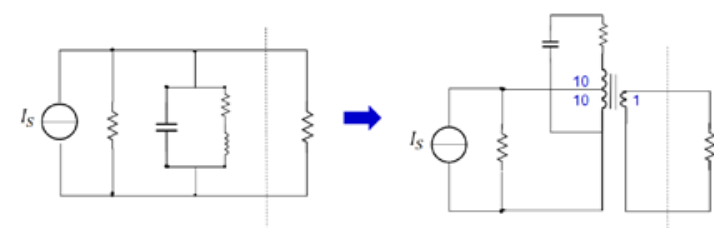

The source resistance vill increase 4. The load resistance will increase 400 tiees

Fig. 16. Reduce the impact of the $\mathrm{Q}$ value

\section{FURTHER THINKING}

What other resonance applications are there in our life? What other areas can resonance be applied in [5, 6]? The previous study tells us that the equivalent impedance is 0 in series resonance, and the equivalent impedance is infinite in parallel resonance. And can we further apply this knowledge to the extraction of useful signals and the suppression of noise?

\section{CONCLUSIONS}

This course explained the theory and application of resonance with the examples in real life and received the praise of the students. In the following courses, we will continue to explain the theory with practice and apply the knowledge in books to practice. Let us learn the circuit course happily.

\section{REFERENCES}

[1] Chen Xiyou. Circuit theory course. Higher Education Press, 2013.8.

[2] C.K.Alexander M.N.O.Sadiku,translated by Yu Xinjie. Fundamentals of Electric Circuits, 5E. Machinery Industry Press. 2013.2.

[3] Qiu Guanyuan, Luo Xianjue. Electric circuits, 5th Ed.. Higher Education Press, 2006.5.

[4] James W. Nilsson, Susan Riedel. Electric circuits, 10th Ed., Electronic Industry Publishing House. 2015.3.

[5] Dai Jing. Study on Capacitor Charging Power Supply Based on the Principle of the Series Resonant. Dissertation of Huazhong University of Science and Technology. 2014.

[6] J. Koscelnik etc.. Design and Simulation of Multi-element Resonant LCTLC Converter with HF Transformer. ELEKTRO. 2014: 307 - 311. 